\title{
Development of a Digital Hypsometric Model of the Territory
}

\author{
Rans Aliev ZH* \\ Institute of Soil and Agro chemistry of the nas of Azerb, Azerbaijan \\ Received: June 15, 2018; Published: July 03, 2018 \\ *Corresponding author: Rans Aliev ZH, Institute of Soil and Agro chemistry of the nas of Azerb, Azerbaijan
}

\begin{abstract}
One of the foundations of spatial analysis methods based on the digital hypsometric model is the development of a map of slope angles. It has received wide application-from morphogenetic and geological-engineering problems to agrarian and territorial planning. The Arc Map program provides an opportunity for rapid preparation of this type of maps based on the raster hypsometric model of the territory in the example of the Republic of Azerbaijan, where it is considered the technique of creating a CTM with the use of the Topo to Raster method, which allows to develop raster layers of CMG on the basis of isolines.
\end{abstract}

Keywords: Maps; Relief; Analog Maps; Terrestrial Landscapes; Isogyps Vectorization; Relief; Factor; Erosion Processes; Topo; Territory; Hypsometric Model

\section{Introduction}

One of the most commonly used interpolation techniques is the digital hypsometric model (CGM). A model of this type is a discrete presentation of the terrain, where each point is assigned its absolute height. In the next part of the task we will talk about the technique of creating a CMM using the Topo to Raster method. This method allows us to develop raster layers of CMG on the basis of isolines [1]. Isohypses are the most common methods of mapping the terrain on analog maps (Figure 1).

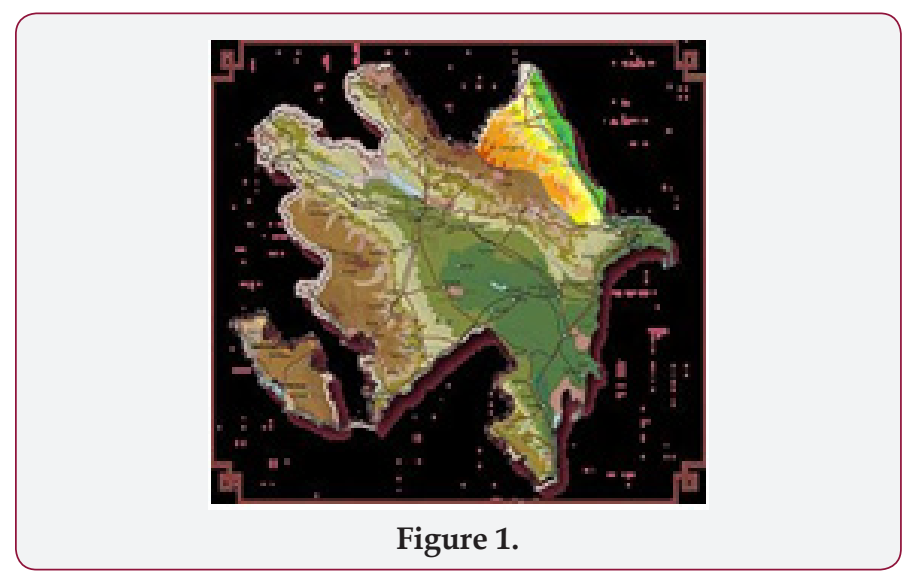

\section{Course of Research and Discussion of Materials}

The application of the Topo to Raster method requires the previous Vectorization of isohypses, with each isoline having an attribute with an absolute height. The method of Topo to Raster proposed here is based on the conditions that erosion processes involving current waters are the main relief-forming factor for most types of terrestrial landscapes. Proceeding from this [2], in the relief of this territory certain skeletal forms-lines of ridges or river valleys are clearly expressed. For most types of landscapes, relatively shallow depressions are relatively rare (with the exception, for example, of a young postglacial relief).

The Topo to Raster method, using iterative techniques, generates a hypsometric model that incorporates the integrity of the surface runoff.

At the first stage, based on the curvature of the lines, a set of skeleton elements (lines of ridges and river valleys) is displayed, and then high-level data are generated for discrete space points. If the cavity grooves cannot be determined in a separate layer, the algorithm will strive for such alignment of the interpolated surface in order to remove all drain less zones from it (on the principle of providing a continuous runoff surface). In addition to layers with isohypes and in-depth depressions, the CMM can be detailed on the basis of additional information layers. They can be, for example, layers with high-rise pickets. The method also makes it possible to use layers with hydrograph of the territory where there are rivers (a linear layer) and lakes (a polygonal layer). The possibilities of this method can be traced by developing a digital hypsometric model of the territory of Azerbaijan [3].

a) Create a new Arc Map project. 
b) For the development of the CTMM, at least three information layers are required that display isohypses, high-altitude pickets and a hydro graphic network.

c) Select the Show / Hide Arc Toolbox Window on the standard toolbar.

d) In the toolbox window (Toolbox) select Spatial Analyst Tool, then Interpolation.

e) From the list of tools choose the method Topo to Raster. The window shown in (Figure 2) appears.

f) Using the Input feature data list, select the information layers required for the CMF creation, which will be added to the table below.

g) After that, in each layer it is necessary to parameterize the type of objects (for example, isohypses or rivers) and in the layer "high-rise picket" the attribute that values the absolute height of the territory [4]. Since Topo to Raster uses primarily isohypses, the program automatically works so that the added layer is represented by contours (Figure 2).

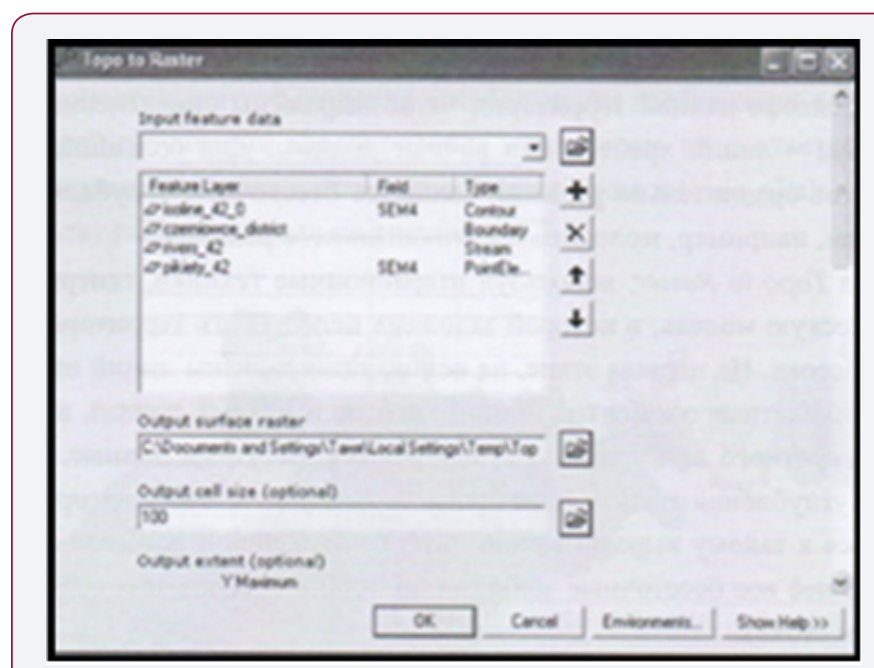

Figure 2: Tope to Raster window.

Hence follows the appearance in the table of the graph Type next to the name of the value of Contour. In the Field column, an attribute with absolute surface height values is denoted.

h) From the lists of the Input feature column, select the layer with high pickets. This layer will help to detail the hypsometric model of the territory. Notice that in the Type column. After adding a layer to the table, the Contour value appears.

i) In the table, click on the Contour graph next to the name of the layer with high-rise pickets. This will open the list with the values available for this column.

j) In a similar way, in the Field column, the attribute with the absolute height values of the picket is indicated.

k) Using the Input feature field, we add to the analysis a layer of the river. In the table near the name of the river, change the value of the Type column to Stream.

l) On completion we add to the analysis the layer with the boundaries of the territory was investigated

m) Go to the graph Qutput surfact raster; select the localization and the name of the source file. The length of the name should not exceed 10 characters, so it is better to use the Latin alphabet.

n) In the Qutputcell size column, we specify the measurement of the initial raster, expressed in units of the map, or the resolving power of the method. This value depends on several factors. The main goal is to create a CMM, and hence the required accuracy of the display.

o) For other parameters of the method, we leave the automatic values. Click OK to start the settlement procedure. Dependent on the computing power of the computer, it should last no more than a few and less than 20 minutes. After completing the calculations, the original layer will be automatically added to the map image. Since the raster layers automatically open under the vector layers, to close the output map, close the vector layers in the layer manager window.

p) Change the layer's display mode to solid (stretched) and select the type of color corresponding to the hypsometric images. The final effect of this work is seen in (Figure 3).

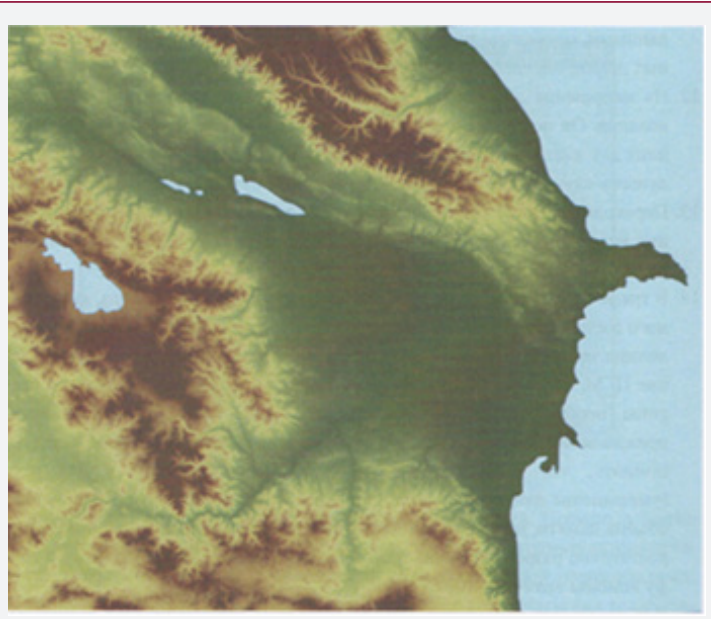

Figure 3: The digital hypsometric model of Azerbaijan, created by the method of Topo to Raster.

q) Finally, you can mark the Show Map Tips option in the window with the properties of the layer. Due to this, it is possible to track the change in the absolute heights of the surface by moving the cursor over the map [5].

The finished digital hypsometric model of the territory can be used for further spatial analysis, which will be discussed in the next section. In the end, we try to improve the appearance of the raster map, for greater readability. We use the shadow effect for this Open the Spatial Analyst toolbar [6].

a) In the Spatial Analyst menu, select Surfact Analyst, then Hilishade the window shown in (Figure 4) Dialog window Hilishade

b) In the Hilishade window, enter the name of the surface in the Input Surfact field, from which the shadow effect will be created. Since there is only one raster layer in the project for now, the program automatically inserts it into this window. 


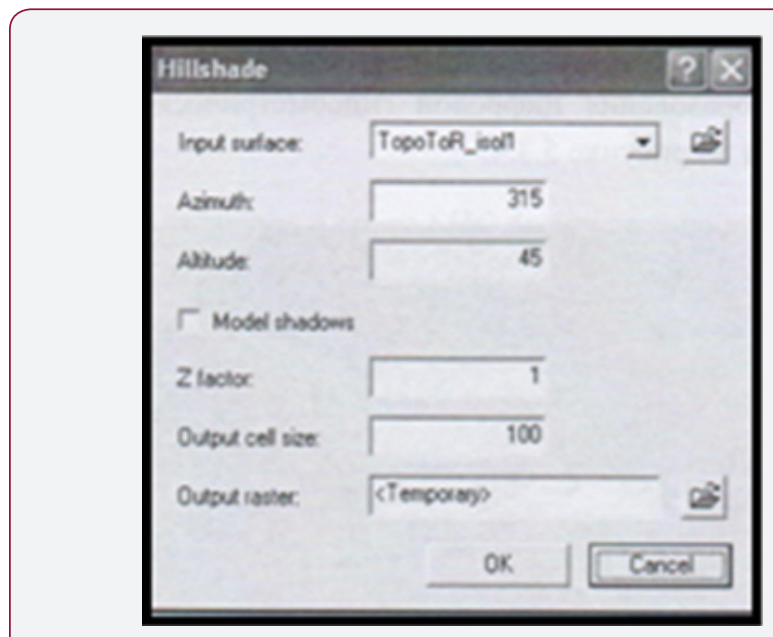

Figure 4: Dialog window Hilishade.

c) The following two text boxes allow you to outline directions and angles of exposure. We arrange automatic values in both graphs, but theoretically in the Azimuth column you can enter values from 0 to 359, and in the graph Altitude numbers from 0 to 90 .

d) The graph $\mathrm{Z}$ factor allows determining the vertical excess for the shadow effect. An automatically inserted value of 1 makes this excess impossible. Values greater than 1 give an opportunity to strengthen the shadow effect, which is a desire for a territory with a monotonous relief.

e) The graph Output cell size performs a similar role, as in the case of the Topo to Raster method. When determining the permissiveness of the effective layer, it is necessary to take the value of the permissive capacity, which is identical to the value of the primary layers. Therefore, in this column we leave the automatic value.

f) In the Output raster column, enter the localization and the name of the source file, and then select $\mathrm{OK}$, which will lead to the beginning of the calculation procedure. After its end, the resulting layer will be added to the preview.

g) Go to the window with the properties of the layer created and in the Symbology tab change the display mode of Stretched Standard Deviation.

h) The resulting image improves the perception of the terrain [7].

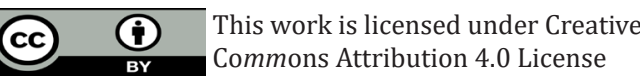

Submission Link: https://biomedres.us/submit-manuscript.php
The effect can be deepened by adding a gamut of hypsometric colors. To do this, in the window with the properties of the layer, go to the Display tab and then enter the value 35 in the transparent column and click OK [8]. The transformation effect of the digital hypsometric model of Azerbaijan is shown in (Figure 5).

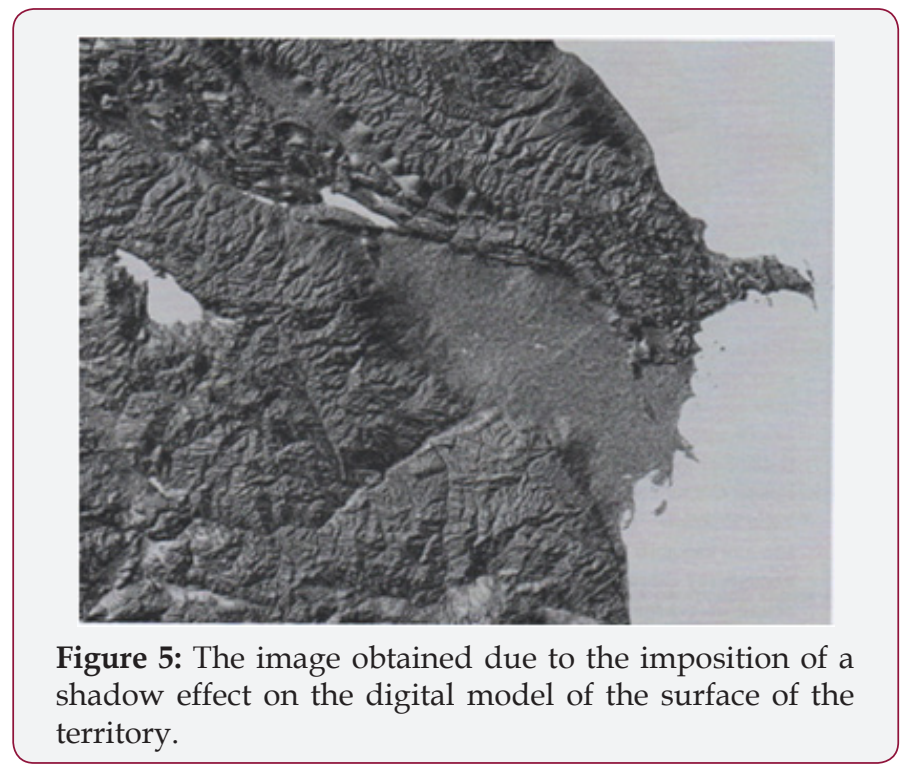

\section{References}

1. BH Aliev, Aliev ZH (2001) Zoning of territory of Azerbaijan Republic on choosing advanced irrigation techniques. Monograph, Publishing house "Ziya", Baku, pp. 297.

2. BH Aliev, Aliev ZH (2003) Irrigated agriculture in the mountain and foothill regions of Azerbaijan. Monograph Publishing house "Nurlan Zia EPG Ltd", Baku pp. 330.

3. Aliev ZH (2007) The premises about the most important problem of the agriculture in provision water resource mountain and foothill regions Azerbaijan, Baku, pp. 179-182.

4. Aliev ZH (1999) The premises of the decision of the problems moisture provides agriculture cultures production in mountain and foothill region Azerbaijan. The works SRI Erosions and Irrigations, Baku, pp. 125-129.

5. Berry K (2006) Mar Ayalesis Procedures and Application in GIS Modeling BASIS Press.

6. Bonham Carter GF (1994) Geographic Information Systems for land resources assessment, Clarendon Press, 0xford.

7. DeMers MN (2002) GIS modeling in raster, John Wiley \& Sons.

8. DeMers MN (2005) Fundamentals of Geographic Information Systems, John Wiley \& Sons.

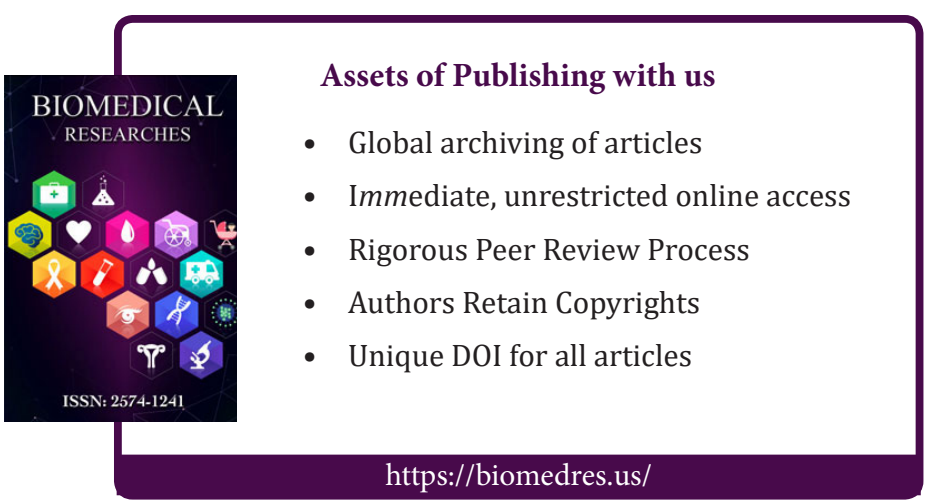

